\title{
Démarche d'estimation des incertitudes de mesure dans un laboratoire de recherche : apport et perspectives - exemple d'un laboratoire de recherche en hydrologie des milieux aquatiques
}

\author{
Carole Beaulieu ${ }^{(1)}$, Gérard Pineau ${ }^{(2)}$, Aurélien Ballu ${ }^{(2)}$, Laurent David ${ }^{(2)}$ et Damien Calluaud ${ }^{(2)}$ \\ (1) LNE Pôle des Eco-Industries - 3, rue Raoul Follereau - 86000 POITIERS \\ (2) INSTITUT Pprime - CNRS - Université de Poitiers - Isea/Ensma \\ UPR CNRS 3346 Bâtiment H2 - Téléport 2 Boulevard Marie \& Pierre Curie 86962 FUTUROSCOPE CHASSENEUIL cedex
}

\begin{abstract}
The design of hydraulic structures for the upstream migration around dams as the vertical slot fishways is very important for the engineers for applying the European Water Directive (2000/60/CE) or the French law (Lema), One of the main parameters for the design is the discharge coefficient. For guaranteeing this coefficient, an evaluation of the uncertainties has been carried out. This approach allows the identification of the different parameters which influence this measurement. Among all the parameters, the water depth and the slope measurements are the major quantities which influence the more the results of the measurements. The evaluation of the uncertainties in laboratory will be extended to the measurements of other quantities.
\end{abstract}

\section{Contexte}

Dans le cadre de la directive Cadre Européenne et de la loi sur l'eau et des milieux aquatiques, la continuité écologique des cours d'eau doit être assurée et de ce fait, un certain nombre de dispositifs permettant d'assurer cette continuité a besoin d'être correctement dimensionné. Dans ce contexte, l'institut Pprime travaille en collaboration étroite avec l'Office National de l'Eau et des Milieux Aquatiques sur le dimensionnement d'ouvrages hydrauliques et des dispositifs de franchissement des poissons tels les passes à poisson. Différentes solutions existent comme les passes naturelles, les passes à ralentisseur ou les passes à fente verticales mais celles-ci demandent toujours un dimensionnement précis afin d'être le plus efficace possible. Pour cela un certain nombre de grandeurs sont indispensables comme les grandeurs géométriques du bassin, les hauteurs d'eau amont et aval, la pente mais également des coefficients qui sont propres à la forme choisie comme la rugosité de fond ou encore le coefficient de débit. Ce dernier coefficient relève d'un intérêt particulier car de sa valeur découle le débit qui transitera dans cet ouvrage et par la même de l'efficacité de la passe. Notre travail consiste donc à fournir aux agences et aux bureaux d'études des coefficients de débits les plus réalistes possible car ils sont indispensables à la conception des ouvrages. Les enjeux financiers sont tels qu'il semble préférable pour ce type de mesure d'adopter une démarche d'évaluation des incertitudes de mesures afin de garantir aux usagers une qualité des résultats et un encadrement des valeurs.

\section{Dimensionnement d'une passe à poissons}

\subsection{Etat des lieux des ouvrages en France}

La Directive Cadre Européenne sur l'Eau établit une politique communautaire dans le domaine de l'eau et de la protection de l'environnement afin que les Etats membres atteignent dans quelques années un objectif de bon état écologique des cours d'eau. L'objectif est, plus particulièrement, la possibilité d'assurer, dans des limites économiques acceptables, la migration de l'ensemble de la faune piscicole tout au long des cours d'eau. Cependant les ouvrages hydrauliques tels que les barrages hydroélectriques, les digues et autres obstacles naturels ou artificiels produisent d'importants changements des caractéristiques de l'écosystème fluvial qui se répercutent spécialement sur les espèces migratrices de poissons. Ces barrières physiques aux mouvements périodiques migratoires des poissons peuvent conduire à la diminution, ou parfois, la disparition complète de certaines espèces. L'aménagement de dispositifs de franchissement permet aux poissons migrateurs de passer un obstacle qui constitue un effet barrière empêchant leur libre circulation entre les biefs amont et aval d'un cours d'eau obstrué, en les attirant en un point de la rivière situé à l'aval de l'obstacle et les incitant à passer en amont passivement par déversement (systèmes de transport et de capture, ascenseurs et écluses) ou activement par la nage, via un canal ou un chenal (passes naturelles, passes à bassins successifs telles les passes à poisson à fentes

carole.beaulieu@lne.fr

This is an Open Access article distributed under the terms of the Creative Commons Attribution License 4.0, which permits unrestricted use, distribution, and reproduction in any medium, provided the original work is properly cited. 
verticales / vertical slot fishway VSF). Les différents dispositifs de franchissement construits depuis une vingtaine d'années en France dans le cadre des différents programmes de protection ou restauration étaient destinés à assurer le passage d'un nombre limité d'espèces-cibles correspondant aux espèces considérées comme migratrices dans le Code de l'Environnement, c'est-à-dire plus particulièrement le saumon, la truite de mer, l'alose et la lamproie, espèces de grandes tailles et aux capacités de nage importantes. Il convient dorénavant de prendre en compte, dans le concept de restauration de la connectivité biologique des cours d'eau, l'ensemble des espèces, dont certaines sont de petites tailles et possèdent des capacités de nage limitées. Cela passe par la conception de dispositifs multi-spécifiques et par l'adaptation des VSFs existantes à ces petites espèces. Cette finalité impose de répondre aux questions, encore ouvertes, concernant les aspects essentiels comme l'efficacité biologique fonctionnelle des installations de migration, que ce soit l'évaluation de l'attractivité des VSFs ou la connaissance du comportement des poissons au sein des bassins des VSFs. Les connaissances dans ces domaines sont en augmentation, mais encore rares. Des directives officielles (BfG, 2010 ; DWA, 2010 ; GHAAPPE, 2008 ; CETMEF, VNF, 2008 ; GHAAPPE, 2006 ; Gough et al., 2012) donnent des recommandations, mais une comparaison avec l'état des connaissances scientifiques actuelles est nécessaire.

\subsection{Paramètre principal de dimensionnement : la puissance dissipée}

Dans le dimensionnement des passes à bassin et plus spécifiquement des VSFs, les propriétés hydrodynamiques du système doivent correspondre aux capacités de nage des espèces de poissons pour lesquelles le système est destiné. Les principaux critères de dimensionnement pris en considération sont la différence de hauteur maximale entre les bassins $(\Delta \mathrm{h})$ et la puissance volumique dissipée $\mathrm{P}_{\mathrm{V}}$. La chute entre les bassins, qui détermine la vitesse maximale dans les bassins, est définie par rapport l'espèce cible définie. Elle peut varier de moins de $10 \mathrm{~cm}$ (pour les VSFs spécialement conçues pour les petites espèces de taille $<300 \mathrm{~mm}$ ) à plus de 30 $\mathrm{cm}$ (pour la truite ou grandes espèces diadromes comme le saumon ou la truite de mer, de taille $>300 \mathrm{~mm}$ ). La puissance dissipée volumique $\left(\mathrm{Wm}^{-3}\right)$ est, quant à elle, un indicateur général du niveau d'agitation turbulente dans les bassins. Ce critère communément utilisé et purement empirique fondé sur les valeurs de $P_{V}$ a été proposé dans les années 1980 (Bell, 1986) où il n'y avait pas de moyens techniques de mesure des caractéristiques de la turbulence dans les bassins. Une valeur de $250 \mathrm{Wm}^{-}$ ${ }^{3}$ est généralement considérée comme la limite supérieure pour les salmonidés. Les niveaux inférieurs sont recommandés $\left(150 \mathrm{Wm}^{-3}\right)$ pour l'alose et les espèces de la rivière rhéophiles (Larinier et al . 2002).

\subsection{Paramètre discriminent : le coefficient de débit}

De par le choix de l'espèce cible pour lequel la VSF est destinée, les dimensions des bassins sont déterminées par des valeurs seuils de $\Delta \mathrm{h}$ et $\mathrm{P}_{\mathrm{v}}$, en fonction du débit circulant dans l'installation. La longueur de l'installation est, quant à elle, définie par rapport au choix de la valeur de $\Delta \mathrm{h}$ et de la pente à franchir. Les paramètres $\mathrm{L}, \mathrm{Q}, \Delta \mathrm{h}$ et $P_{V}$ étant fixés, le volume d'eau $V$ dans les bassins est donc obtenu. Il est alors nécessaire de connaitre la relation existante entre la largeur du bassin (B), et la hauteur d'eau dans les bassins (Z), pour déterminer la hauteur d'eau minimale des bassins en fonction de leur largeur. Pour cela, dans la pratique, la largeur des bassins est fixée puis la hauteur d'eau sur la fente (la charge sur la fente) est estimée en utilisant un coefficient empirique issu de mesures expérimentales, le coefficient de débit. Ce dernier caractérise la perte de charge liée au passage de l'eau d'un bassin à un autre. En conséquence, le dimensionnement des bassins de la passe à poissons peut être réalisé grâce à un seul paramètre issu de mesures expérimentales : le coefficient de débit $\left(\mathrm{C}_{\mathrm{d}}\right)$. Il est donné par l'expression suivante (sans dimension) :

$$
C_{d}=\frac{Q}{b\left(Z_{0}+\frac{L}{2} \sin \alpha-h_{s}\right) \sqrt{2 g\left(Z_{0}-Z+L \sin \alpha\right)}}
$$

Avec :

b la largeur de la fente (m)

$\alpha$ l'inclinaison du bassin (pente $s$ )

$\mathrm{h}_{\mathrm{s}} \quad$ la hauteur de seuil (m)

L la longueur du bassin (m)

$\mathrm{Z}$ la hauteur d'eau à mi-bassin $(\mathrm{m})$

$\mathrm{Z}_{0}$ la hauteur d'eau à mi-bassin du bassin amont $(\mathrm{m})$

$\mathrm{g}$ l'accélération de la pesanteur $\left(\mathrm{m} / \mathrm{s}^{2}\right)$

Q le débit volumique $\left(\mathrm{m}^{3} / \mathrm{s}\right)$

\section{Présentation du dispositif expérimental du laboratoire P'}

\subsection{Présentation du dispositif}

Le dispositif expérimental utilisé lors des essais a été entièrement conçu et réalisé au sein de l'Institut Pprime. Il modélise une passe à poissons à fentes verticales. Son dimensionnement a été déterminé en se référant aux données moyennes des passes à fentes verticales françaises à partir d'un critère de similitude de Froude ${ }^{1}$ basé sur la hauteur d'eau à mi-bassin $\mathrm{Z}$.

L'échelle géométrique retenue pour le dispositif expérimental est de $1 / 4$, celles des vitesses et des puissances volumiques de $1 / 2$, et celle des débits de $1 / 32$. La passe à poissons à l'échelle $1 / 4$ est présentée sur la Figure 1.

Le dispositif fonctionne en boucle fermée. Une pompe centrifuge permet d'aspirer l'eau dans des bacs de

\footnotetext{
${ }^{1}$ Le nombre de Froude est le rapport des forces d'inerties sur les forces de pesanteurs.
} 
rétentions avals et la restitue dans un bac de rétention amont. Un débitmètre, utilisé pour mesurer le débit de la pompe, permet par l'intermédiaire du variateur de la pompe, de réguler le débit autour de la valeur fixée par l'utilisateur. L'eau contenue dans le bac de rétention amont s'écoule alors par gravité à travers 5 bassins modélisant la passe à poissons. Ces bassins, réalisés en matière transparente (PMMA) permettent aussi de visualiser l'écoulement par l'intermédiaire de techniques laser et vidéos.

Un système de guillotine installé sur crémaillère à l'extrémité de la passe à poissons permet d'équilibrer les hauteurs de chutes entre les bassins.

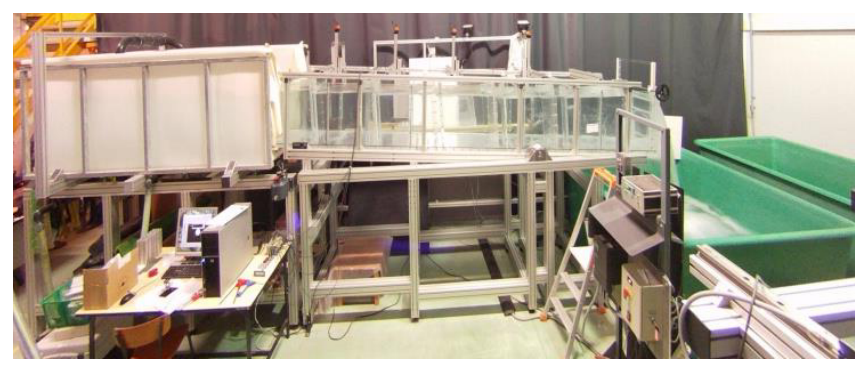

Figure 1 : Dispositif expérimental de l'Institut Pprime

\subsection{Plage des paramètres}

Le modèle de passe à poissons à fentes verticales (échelle 1/4) du laboratoire est en partie modulable. Il est possible de faire varier la pente à l'aide d'un vérin mais aussi la largeur des bassins en translatant une paroi amovible. Les plages de variations des paramètres sont mentionnées au sein du tableau ci dessous.

Tableau 1 :

Caractéristiques du modèle de passe à poissons de l'Institut Pprime

\begin{tabular}{|c|c|c|}
\hline \multirow{4}{*}{$\begin{array}{c}\text { Caractéristiques } \\
\text { générales du } \\
\text { modèle }\end{array}$} & $\begin{array}{c}\text { Similitude } \\
\text { géométrique }\end{array}$ & $\begin{array}{c}\text { Échelle vitesses } \\
\text { \& puissances } \\
\text { volumiques }\end{array}$ \\
\cline { 2 - 3 } & Échelle des débits & $1 / 4$ \\
\hline \multirow{2}{*}{$\begin{array}{c}\text { Caractéristiques } \\
\text { géométriques } \\
\text { d'un bassin }\end{array}$} & Largeur fente & $\mathrm{b}=0,075 \mathrm{~m}$ \\
\cline { 2 - 3 } & Longueur bassin & $\mathrm{L}=0,75 \mathrm{~m}$ \\
\cline { 2 - 3 } & Largeur bassin & $0,425 \mathrm{~m} \leq \mathrm{B} \leq 0,675 \mathrm{~m}$ \\
\cline { 2 - 3 } & Hauteur bassin & $\mathrm{h}=0,55 \mathrm{~m}$ \\
\cline { 2 - 3 } & Pente $(\%)$ & $5 / 7,5 / 10 / 12,5 / 15$ \\
\hline
\end{tabular}

\subsection{Moyens de mesure associés}

Le dimensionnement des bassins d'une passe à poissons est défini à partir d'un seul paramètre : le coefficient de débit (\$2.3). Pour calculer ce coefficient, il est nécessaire de mesurer la hauteur d'eau au milieu de chaque bassin. Des mesures, sans contact, ont donc été réalisées par des sondes acoustiques positionnées au centre de chaque bassin. Ces sondes acoustiques utilisent des ultrasons pour mesurer la distance séparant la sonde de la cible. Leurs caractéristiques sont recensées dans le tableau 2.
Tableau 2 : Caractéristiques des sondes acoustiques

\begin{tabular}{|l|l|}
\hline Marque & Microsonic \\
\hline Type & Mic $+25 /$ IU/TC \\
\hline Reproductibilité & $+/-0,15 \%$ \\
\hline
\end{tabular}

Les sondes de hauteurs utilisées ont une gamme de mesure comprise entre $30 \mathrm{~mm}$ et $250 \mathrm{~mm}$ (Figure 2).

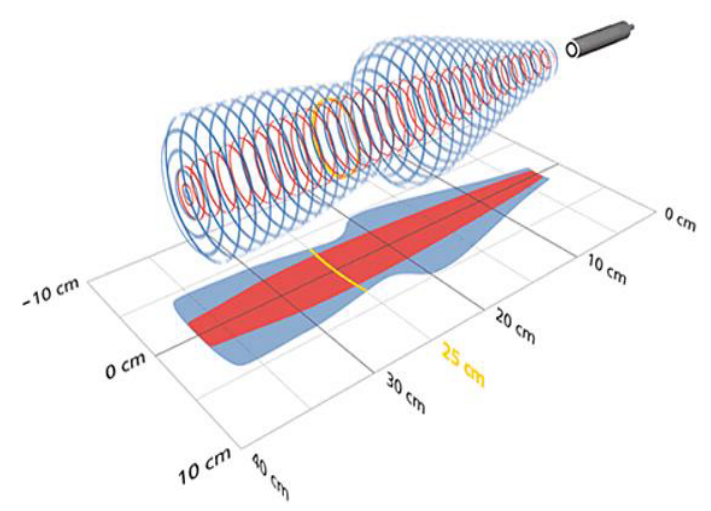

Figure 2 : Plage de détection des sondes acoustiques

Les sondes ont été disposées au milieu des quatre premiers bassins à $\mathrm{L} / 2$ et $\mathrm{B} / 2$ perpendiculairement au radier (Figure 3).

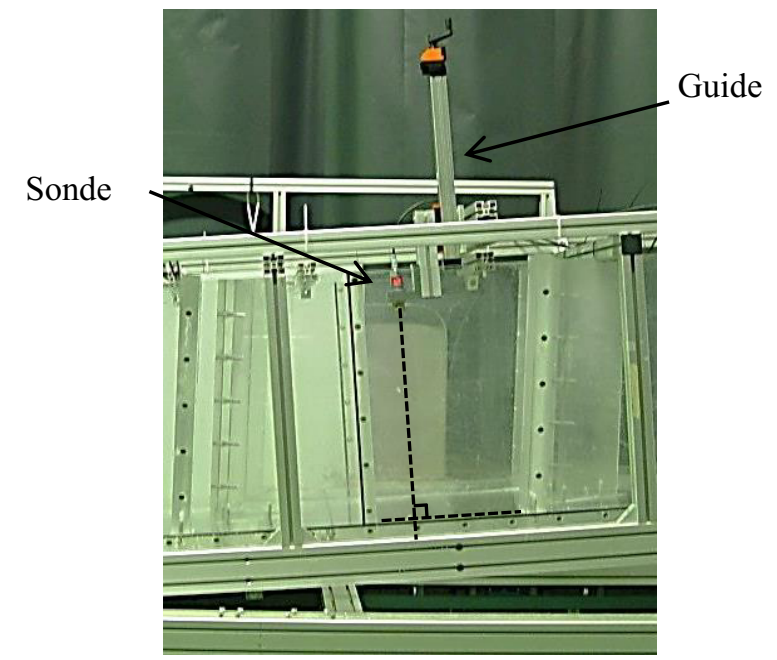

Figure 3 : Fixation des sondes acoustiques dans les bassins

\section{Détermination des paramètres de dimensionnement et estimation des incertitudes associées}

\subsection{Largeur de la fente (b)}

Les fentes correspondent à des zones de passage entre deux déflecteurs et sont délimitées par deux plans parallèles. La largeur nominale des fentes est de $75 \mathrm{~mm}$. 


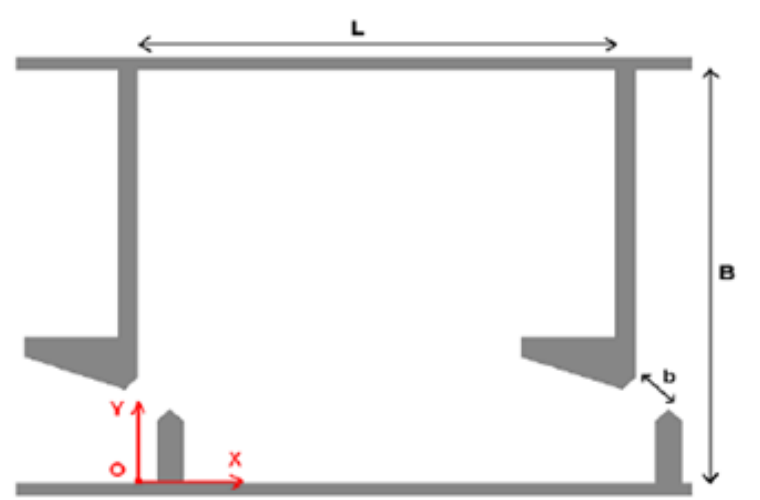

Figure 4 : Schéma de représentation des fentes

Cette largeur est mesurée avec un jeu de cales étalons pour différentes hauteurs et pour toutes les fentes.

La largeur moyenne est de 74,83 $\mathrm{mm}$.

L'incertitude-type sur la largeur de la fente tient compte des performances métrologiques des cales, du défaut de géométrie des fentes et des variations entre les fentes, elle est de $0,65 \mathrm{~mm}$., soit $0,86 \%$.

\subsection{Pente (s) ou inclinaison ( $\alpha$ ) du bassin}

La pente est plus généralement utilisée pour exprimer l'inclinaison du bassin mais c'est l'angle qui intervient dans l'expression du coefficient de débit.

Tableau 3 : Correspondance entre la pente (s) et l'inclinaison ( $\alpha$ )

\begin{tabular}{|l|l|l|l|l|l|}
\hline Pente du bassin $(\mathrm{s})$ & $5 \%$ & $7,5 \%$ & $10 \%$ & $12,5 \%$ & $15 \%$ \\
\hline Inclinaison du bassin $(\alpha)$ & $2,86^{\circ}$ & $4,29^{\circ}$ & $5,71^{\circ}$ & $7,12^{\circ}$ & $8,53^{\circ}$ \\
\hline
\end{tabular}

La pente du bassin est ajustée à l'aide de cales. Elle est mesurée avec un inclinomètre dont l'EMT est de $0,2^{\circ}$.

Les inclinaisons moyennes sont de $3,07^{\circ}, 4,47^{\circ}, 5,90^{\circ}$, $7,37^{\circ}$ et $8,73^{\circ}$.

L'incertitude-type sur l'inclinaison tient compte des performances métrologiques de l'inclinomètre et du défaut d'inclinaison du radier, elle est donnée dans le tableau ci-dessous en fonction de l'inclinaison :

Tableau 4 : Incertitude-type relative sur l'inclinaison du bassin ( $\alpha$ ) en fonction de l'inclinaison du bassin $(\alpha)$

\begin{tabular}{|l|l|l|l|l|l|}
\hline Inclinaison du bassin $(\alpha)$ & $2,86^{\circ}$ & $4,29^{\circ}$ & $5,71^{\circ}$ & $7,12^{\circ}$ & $8,53^{\circ}$ \\
\hline $\begin{array}{l}\text { Incertitude-type relative } \\
\text { sur 1'inclinaison du bassin } \\
(\alpha)\end{array}$ & $5,43 \%$ & $3,73 \%$ & $2,65 \%$ & $2,26 \%$ & $1,52 \%$ \\
\hline
\end{tabular}

\subsection{Hauteur du seuil $\left(h_{s}\right)$}

La hauteur du seuil est dimensionnée par rapport à la largeur de la fente (b). Les trois hauteurs de seuil

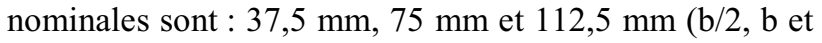
$3 \mathrm{~b} / 2$ ).

Cette hauteur est mesurée avec un pied à coulisse de capacité $150 \mathrm{~mm}$ à $0,01 \mathrm{~mm}$ dont l'EMT est connue (conforme à la norme NF E 11-091 : 2013).

Les hauteurs de seuil moyennes sont de $37,151 \mathrm{~mm}$, $75,401 \mathrm{~mm}$ et $112,971 \mathrm{~mm}$.
L'incertitude-type sur la hauteur du seuil tient compte des performances métrologiques du pied à coulisse et des variations entre les seuils, elle est de $0,13 \mathrm{~mm}$ soit $0,35 \%$ pour $37,5 \mathrm{~mm}, 0,07 \mathrm{~mm}$ soit $0,10 \%$ pour $75 \mathrm{~mm}$ et 0,09 $\mathrm{mm}$ soit $0,08 \%$ pour $112,5 \mathrm{~mm}$.

\subsection{Longueur du bassin (L)}

Tous les bassins ont une longueur nominale de $750 \mathrm{~mm}$. Cette longueur est mesurée avec un mètre à ruban de $2 \mathrm{~m}$ de classe II (EMT de 0,7 mm).

La longueur moyenne des bassins est de 754,64 mm.

L'incertitude-type sur la longueur du bassin tient compte des performances métrologiques du mètre à ruban, du défaut de parallélisme du bassin et des différences de longueur entre les quatre bassins, elle est de $0,75 \mathrm{~mm}$, soit $0,10 \%$.

\subsection{Largeur du bassin (B)}

La largeur du bassin (B) n'apparaît pas dans l'expression $\mathrm{du}$ coefficient de débit $\left(\mathrm{C}_{\mathrm{d}}\right)$ mais est un paramètre de configuration qui fait varier les hauteurs d'eau $\left(Z\right.$ et $\left.Z_{0}\right)$.

\subsection{Hauteur d'eau à mi-bassin $(Z)$}

La hauteur d'eau (Z) n'est pas un paramètre de dimensionnement, elle est mesurée lors de chaque essai. Les mesures sont sans contact et se font à l'aide d'un capteur acoustique. Le capteur est positionné au milieu du bassin au minimum à $30 \mathrm{~mm}$ au-dessus du niveau de l'eau (pour éviter le contact avec l'eau et les projections). Le capteur est un transmetteur 4-20 mA associé à un convertisseur courant/tension et à un voltmètre instrumenté et piloté.

Le capteur est positionné perpendiculairement au radier, la hauteur mesurée doit donc être corrigée car elle ne correspond pas exactement au milieu du bassin comme le montre la Figure 5.

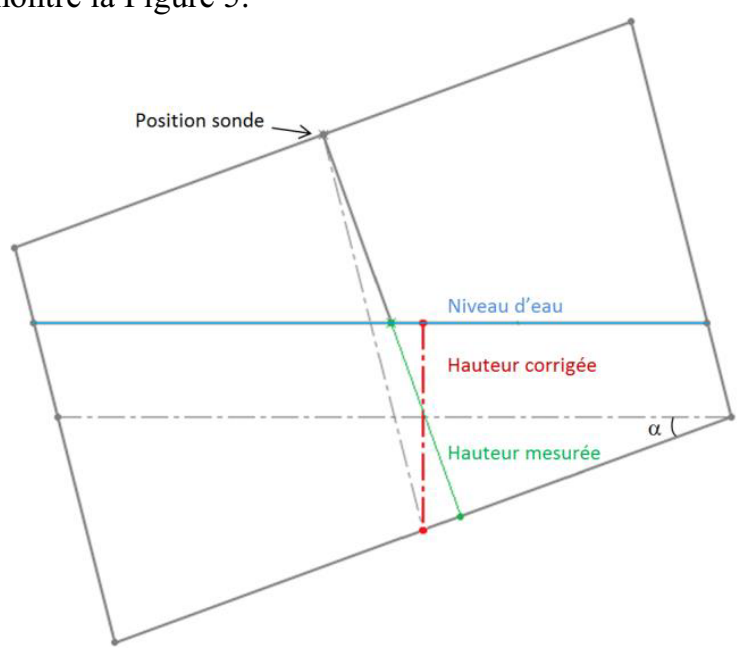

Figure 5 : Illustration de la correction de verticalité de l'installation

Le tableau 5 donne la valeur du terme correctif en fonction de l'inclinaison. 
Tableau 5 : Valeur de la hauteur d'eau à mi-bassin $(Z)$ en fonction de la valeur mesurée $\left(Z_{m e s}\right)$ et de l'inclinaison du bassin $(\alpha)$

\begin{tabular}{|c|c|c|c|c|c|c|}
\hline $\begin{array}{l}\text { Inclinaison } \\
\text { bassin }(\alpha)\end{array}$ & $\mathrm{du}$ & $2,86^{\circ}$ & $4,29^{\circ}$ & $5,71^{\circ}$ & $7,12^{\circ}$ & $8,53^{\circ}$ \\
\hline \multirow{2}{*}{$\begin{array}{l}Z \text { en } \\
\text { fonction de } \\
\text { la valeur } \\
\text { mesurée par } \\
\text { le dispositif } \\
Z=a Z_{\text {mes }}+b\end{array}$} & $\mathrm{a}$ & 0,9987 & 0,9972 & 0,9950 & 0,9923 & 0,9889 \\
\hline & $\begin{array}{c}\mathrm{b} \\
(\mathrm{mm})\end{array}$ & 2,896 & 4,338 & 5,771 & 7,194 & 8,604 \\
\hline
\end{tabular}

Le raccordement métrologique du dispositif de mesure est réalisé à l'aide d'une cale étalon de $350 \mathrm{~mm}$ et d'un pied à coulisse. L'incertitude-type correspondante est de 0,11 $\mathrm{mm}$, ce qui correspond à une incertitude-type relative comprise entre $0,05 \%$ à $0,26 \%$.

La dispersion des mesures dépend de la configuration (inclinaison, débit volumique et hauteur de seuil), le tableau ci-dessous indique les dispersions moyennes observées en fonction de l'inclinaison :

Tableau 6 : Ecart-type moyen relatif sur les mesures de hauteur d'eau à mi-bassin $(Z)$ en fonction de l'inclinaison du bassin $(\alpha)$

\begin{tabular}{|l|l|l|l|l|l|}
\hline Inclinaison du bassin $(\alpha)$ & $2,86^{\circ}$ & $4,29^{\circ}$ & $5,71^{\circ}$ & $7,12^{\circ}$ & $8,53^{\circ}$ \\
\hline $\begin{array}{l}\text { Ecart-type moyen sur les } \\
\text { mesures de hauteur d'eau } \\
\text { à mi-bassin (\%) }\end{array}$ & 1,36 & 2,30 & 2,52 & 3,13 & 3,74 \\
\hline
\end{tabular}

L'incertitude-type sur la hauteur d'eau à mi-bassin tient compte des performances métrologiques du dispositif de mesure, de la dispersion des mesures en fonction des différentes configurations (positionnement, facteur humain) et du terme correctif de verticalité. Elle est donnée dans le tableau ci-dessus en fonction de l'inclinaison:

Tableau 7 : Incertitude-type relative sur la hauteur d'eau à mibassin $(Z)$ en fonction de l'inclinaison du bassin $(\alpha)$

\begin{tabular}{|l|l|l|l|l|l|}
\hline Inclinaison du bassin $(\alpha)$ & $2,86^{\circ}$ & $4,29^{\circ}$ & $5,71^{\circ}$ & $7,12^{\circ}$ & $8,53^{\circ}$ \\
\hline $\begin{array}{l}\text { Incertitude-type relative } \\
\text { sur la hauteur d'eau à mi- } \\
\text { bassin }(\mathrm{Z})\end{array}$ & 1,38 & 2,30 & 2,52 & 3,13 & 3,74 \\
\hline
\end{tabular}

\subsection{Hauteur d'eau à mi-bassin du bassin amont} $\left(Z_{0}\right)$

Même détermination que pour la hauteur d'eau à mibassin :

Tableau 8 : Incertitude-type relative sur la hauteur d'eau à mi-bassin du bassin amont $\left(Z_{0}\right)$ en fonction de l'inclinaison du bassin $(\alpha)$

\begin{tabular}{|l|l|l|l|l|l|}
\hline Inclinaison du bassin $(\alpha)$ & $2,86^{\circ}$ & $4,29^{\circ}$ & $5,71^{\circ}$ & $7,12^{\circ}$ & $8,53^{\circ}$ \\
\hline $\begin{array}{l}\text { Incertitude-type relative } \\
\text { sur la hauteur d'eau à mi- } \\
\text { bassin du bassin amont } \\
\left(\mathrm{Z}_{0}\right)\end{array}$ & 1,38 & 2,30 & 2,52 & 3,13 & 3,74 \\
\hline
\end{tabular}

\subsection{Accélération de la pesanteur $(g)$}

La norme NF X 02-011: 1974 indique l'accélération de la pesanteur en fonction de la latitude et de l'altitude d'un lieu.

Le dispositif expérimental du laboratoire P' se trouve à une latitude de $46,66^{\circ}$ et à une altitude de $100 \mathrm{~m}$, l'accélération de la pesanteur locale (g) est donc de $9,8073 \mathrm{~m} / \mathrm{s}^{2}$ avec une incertitude-type relative de $5.10^{-5}$.

\subsection{Débit volumique (Q)}

Le débit volumique (Q) est compris entre $0,018 \mathrm{~m}^{3} / \mathrm{s}$ et $0,032 \mathrm{~m}^{3} / \mathrm{s}$.

Le débit volumique $(\mathrm{Q})$ est directement mesuré par un débitmètre dont les performances métrologiques sont connues $(\mathrm{EMT}=0,5 \%)$.

L'incertitude-type sur le débit volumique tient essentiellement compte des performances métrologiques du débitmètre, elle est de 0,29\% (l'influence du débit volumique sur la dispersion des mesures de hauteurs étant déjà prise en compte).

\subsection{Coefficient de débit $\left(C_{d}\right)$}

Toutes configurations confondues, le coefficient de débit $\left(C_{d}\right)$ est compris entre 0,6 et 0,9 .

Compte-tenu de l'expression du coefficient de débit $\left(\mathrm{C}_{\mathrm{d}}\right)$, les incertitudes-types sur chaque paramètre sont à convertir afin d'estimer l'incertitude-type composée sur le coefficient de débit.

Les contributions de la largeur de la fente (b), de la hauteur de seuil $\left(h_{\mathrm{s}}\right)$, de la longueur du bassin $(\mathrm{L})$, de l'accélération de la pesanteur ( $\mathrm{g}$ ) et du débit volumique (Q) sont négligeables devant les contributions de l'inclinaison $(\alpha)$ et des hauteurs d'eau à mi-bassin $(Z$ et $\mathrm{Z}_{0}$ ).

L'incertitude élargie est globalement comprise entre 18\% et $30 \%$ toutes configurations confondues, elle augmente avec le débit volumique (Q). La contribution de l'inclinaison $(\alpha)$ diminue avec la pente alors que les contributions des hauteurs d'eau $\left(Z\right.$ et $\left.Z_{0}\right)$ augmentent. Le paramètre le plus influent est la hauteur d'eau à mi-bassin du bassin amont $\left(Z_{0}\right)$ qui peut représenter jusqu'à $75 \%$.

Tableau 9 : Contribution des différents paramètres lors de la détermination du coefficient de débit $(\mathrm{Cd})$ pour une pente de $5 \%$

\begin{tabular}{|c|c|c|c|}
\hline \multicolumn{4}{|c|}{ Pente de 5\% } \\
\hline Grandeur & $\begin{array}{l}\text { Incertitude-type } \\
\text { sur la grandeur }\end{array}$ & $\begin{array}{l}\text { Incertitude-type } \\
\text { correspondante } \\
\text { ramenée en } \\
\text { coefficient de } \\
\text { débit (valeur } \\
\text { relative) }\end{array}$ & $\begin{array}{l}\text { Contribution } \\
\text { dans } \\
\text { l'incertitude } \\
\text { totale }\end{array}$ \\
\hline $\mathrm{b}$ & $0,86 \%$ & $8,6.10^{-3}$ & $<1 \%$ \\
\hline$\alpha$ & $5,43 \%$ & $6,4 \cdot 10^{-2}$ à $8,3 \cdot 10^{-2}$ & $20 \%$ à $38 \%$ \\
\hline $\mathrm{h}_{\mathrm{s}}$ & $0,10 \%$ & $<3,2.10^{-4}$ & $<1 \%$ \\
\hline $\mathrm{L}$ & $0,10 \%$ & $<5,8.10^{-4}$ & $<1 \%$ \\
\hline$Z$ & $1,38 \%$ & $6,9 \cdot 10^{-2}$ à $8,6 \cdot 10^{-2}$ & $25 \%$ à $30 \%$ \\
\hline $\mathrm{Z}_{0}$ & $1,38 \%$ & $8,4.10^{-2}$ à $9,2.10^{-2}$ & $37 \%$ à $50 \%$ \\
\hline $\mathrm{g}$ & $0,005 \%$ & $2,5 \cdot 10^{-5}$ & $<1 \%$ \\
\hline $\mathrm{Q}$ & $0,29 \%$ & $2,9.10^{-3}$ & $<1 \%$ \\
\hline \multicolumn{3}{|c|}{$\begin{array}{l}\text { Incertitude élargie sur la détermination du } \\
\text { coefficient de débit } C_{d} \text { pour une pente de } 5 \% \\
(k=2)\end{array}$} & de $26 \%$ à $30 \%$ \\
\hline
\end{tabular}


Tableau 10 : Contribution des différents paramètres lors de la détermination du coefficient de débit $(\mathrm{Cd})$ pour une pente de $7,5 \%$

\begin{tabular}{|l|l|l|l|}
\hline \multicolumn{3}{|c|}{ Pente de 7,5\% } \\
\hline Grandeur & $\begin{array}{l}\text { Incertitude-type } \\
\text { correspondante } \\
\text { ramenée en } \\
\text { sur la grandeur } \\
\text { coefficient de } \\
\text { débit (valeur } \\
\text { relative) }\end{array}$ & $\begin{array}{l}\text { Contribution } \\
\text { dans } \\
\text { l'incertitude } \\
\text { totale }\end{array}$ \\
\hline $\mathrm{B}$ & $0,86 \%$ & $8,6.10^{-3}$ & $<1 \%$ \\
\hline$\alpha$ & $3,73 \%$ & $2,1.10^{-2}$ à $6,0.10^{-2}$ & $2 \%$ à $25 \%$ \\
\hline $\mathrm{h}_{\mathrm{s}}$ & $0,10 \%$ & $<2,2.10^{-4}$ & $<1 \%$ \\
\hline $\mathrm{L}$ & $0,10 \%$ & $<6,4.10^{-4}$ & $<1 \%$ \\
\hline $\mathrm{Z}$ & $2,30 \%$ & $6,2.10^{-2}$ à $9,2.10^{-2}$ & $25 \%$ à $38 \%$ \\
\hline $\mathrm{Z}_{0}$ & $2,30 \%$ & $8,6.10^{-2}$ à $1,1.10^{-1}$ & $50 \%$ à $60 \%$ \\
\hline $\mathrm{G}$ & $0,005 \%$ & $2,5.10^{-5}$ & $<1 \%$ \\
\hline $\mathrm{Q}$ & $0,29 \%$ & $2,9.10^{-3}$ & $<1 \%$ \\
\hline $\begin{array}{l}\text { Incertitude } \\
\text { coefficient de débit } \mathbf{C}_{\mathbf{d}} \text { pour } \\
(\mathbf{k}=\mathbf{2})\end{array}$ & $\begin{array}{l}\text { détermination } \mathbf{d u} \\
\text { dnente de 7,5\% }\end{array}$ & de 22\% à $\mathbf{2 4 \%}$ \\
\hline
\end{tabular}

Tableau 11 : Contribution des différents paramètres lors de la détermination du coefficient de débit $(\mathrm{Cd})$ pour une pente de $10 \%$

\begin{tabular}{|c|c|c|c|}
\hline \multicolumn{4}{|c|}{ Pente de $10 \%$} \\
\hline Grandeur & $\begin{array}{l}\text { Incertitude-type } \\
\text { sur la grandeur }\end{array}$ & $\begin{array}{l}\text { Incertitude-type } \\
\text { correspondante } \\
\text { ramenée en } \\
\text { coefficient de } \\
\text { débit (valeur } \\
\text { relative) } \\
\end{array}$ & $\begin{array}{l}\text { Contribution } \\
\text { dans } \\
\text { l'incertitude } \\
\text { totale }\end{array}$ \\
\hline $\mathrm{B}$ & $0,86 \%$ & $8,6.10^{-3}$ & $<1 \%$ \\
\hline$\alpha$ & $2,65 \%$ & $3,5 \cdot 10^{-2}$ à $4,5 \cdot 10^{-2}$ & $8 \%$ à $23 \%$ \\
\hline $\mathrm{h}_{\mathrm{s}}$ & $0,10 \%$ & $<2,3.10^{-4}$ & $<1 \%$ \\
\hline $\mathrm{L}$ & $0,10 \%$ & $<6,7.10^{-4}$ & $<1 \%$ \\
\hline $\bar{Z}$ & $2,52 \%$ & $4,4.10^{-2}$ à $6,7.10^{-2}$ & $23 \%$ à $32 \%$ \\
\hline $\mathrm{Z}_{0}$ & $2,52 \%$ & $7,0.10^{-2}$ à $9,3 \cdot 10^{-2}$ & $54 \%$ à $60 \%$ \\
\hline $\mathrm{G}$ & $0,005 \%$ & $2,5 \cdot 10^{-5}$ & $<1 \%$ \\
\hline $\mathrm{Q}$ & $0,29 \%$ & $2,9.10^{-3}$ & $<1 \%$ \\
\hline \multicolumn{3}{|c|}{$\begin{array}{l}\text { Incertitude élargie sur la détermination du } \\
\text { coefficient de débit } C_{d} \text { pour une pente de } 10 \% \\
(k=2)\end{array}$} & de $20 \%$ à $22 \%$ \\
\hline
\end{tabular}

Tableau 12 : Contribution des différents paramètres lors de la détermination du coefficient de débit $(\mathrm{Cd})$ pour une pente de $12,5 \%$

\begin{tabular}{|c|c|c|c|}
\hline \multicolumn{4}{|c|}{ Pente de $12,5 \%$} \\
\hline Grandeur & $\begin{array}{l}\text { Incertitude-type } \\
\text { sur la grandeur }\end{array}$ & \begin{tabular}{|l} 
Incertitude-type \\
correspondante \\
ramenée en \\
coefficient de \\
débit (valeur \\
relative) \\
\end{tabular} & $\begin{array}{l}\text { Contribution } \\
\text { dans } \\
\text { l'incertitude } \\
\text { totale }\end{array}$ \\
\hline $\mathrm{b}$ & $0,86 \%$ & $8,6.10^{-3}$ & $<1 \%$ \\
\hline$\alpha$ & $2,26 \%$ & $3,7.10^{-2}$ à $4,0.10^{-2}$ & $7 \%$ à $20 \%$ \\
\hline $\mathrm{h}_{\mathrm{s}}$ & $0,10 \%$ & $<2,9 \cdot 10^{-4}$ & $<1 \%$ \\
\hline $\mathrm{L}$ & $0,10 \%$ & $<3,2.10^{-4}$ & $<1 \%$ \\
\hline$Z$ & $3,13 \%$ & $4,0.10^{-2}$ à $7,5.10^{-2}$ & $20 \%$ à $30 \%$ \\
\hline$Z_{0}$ & $3,13 \%$ & $7,1.10^{-2}$ à $8,1.10^{-2}$ & $60 \%$ à $63 \%$ \\
\hline $\mathrm{g}$ & $0,005 \%$ & $2,5 \cdot 10^{-5}$ & $<1 \%$ \\
\hline Q & $0,29 \%$ & $2,9 \cdot 10^{-3}$ & $<1 \%$ \\
\hline \multicolumn{3}{|c|}{$\begin{array}{l}\text { Incertitude élargie sur la détermination du } \\
\text { coefficient de débit } C_{d} \text { pour une pente de } 12,5 \% \\
(k=2)\end{array}$} & de $20 \%$ à $28 \%$ \\
\hline
\end{tabular}

Tableau 13 : Contribution des différents paramètres lors de la détermination du coefficient de débit $(\mathrm{Cd})$ pour une pente de $15 \%$

\begin{tabular}{|c|c|c|c|}
\hline \multicolumn{4}{|c|}{ Pente de 15\% } \\
\hline Grandeur & $\begin{array}{l}\text { Incertitude-type } \\
\text { sur la grandeur }\end{array}$ & $\begin{array}{l}\text { Incertitude-type } \\
\text { correspondante } \\
\text { ramenée en } \\
\text { coefficient de } \\
\text { débit (valeur } \\
\text { relative) }\end{array}$ & $\begin{array}{l}\text { Contribution } \\
\text { dans } \\
\text { l'incertitude } \\
\text { totale }\end{array}$ \\
\hline $\mathrm{b}$ & $0,86 \%$ & $8,6 \cdot 10^{-3}$ & $<1 \%$ \\
\hline$\alpha$ & $1,52 \%$ & $2,6.10^{-2}$ à $2,8.10^{-2}$ & $5 \%$ à $12 \%$ \\
\hline $\mathrm{h}_{\mathrm{s}}$ & $0,10 \%$ & $<4,5.10^{-4}$ & $<1 \%$ \\
\hline $\mathrm{L}$ & $0,10 \%$ & $<7,1.10^{-4}$ & $<1 \%$ \\
\hline$Z$ & $3,74 \%$ & $3,7.10^{-2}$ à $7,0.10^{-2}$ & $18 \%$ à $20 \%$ \\
\hline $\mathrm{Z}_{0}$ & $3,74 \%$ & $7,3.10^{-2}$ à $1,1.10^{-1}$ & $70 \%$ à $75 \%$ \\
\hline $\mathrm{g}$ & $0,005 \%$ & $2,5 \cdot 10^{-5}$ & $<1 \%$ \\
\hline $\mathrm{Q}$ & $0,29 \%$ & $2,9.10^{-3}$ & $<1 \%$ \\
\hline \multicolumn{3}{|c|}{$\begin{array}{l}\text { Incertitude élargie sur la détermination du } \\
\text { coefficient de débit } C_{d} \text { pour une pente de } 15 \% \\
(k=2)\end{array}$} & de $18 \%$ à $26 \%$ \\
\hline
\end{tabular}

\section{Conclusions et perspectives}

La conception d'ouvrage de franchissement autour des barrages comme les passes à poisson à fente verticale relève d'un intérêt majeur pour les ingénieurs hydrauliciens afin de satisfaire les législations Française et Européenne sur la continuité écologique. Un des paramètres fondamentaux pour dimensionner cet ouvrage est le coefficient de débit. Afin de garantir les résultats obtenus sur ce coefficient, une démarche d'évaluation des incertitudes de mesure a été entreprise. L'apport de cette démarche est d'identifier dans un premier temps les paramètres influents sur cette mesure. Parmi l'ensemble des paramètres, les contributions de la largeur de la fente (b), de la hauteur de seuil $\left(\mathrm{h}_{\mathrm{s}}\right)$, de la longueur du bassin (L), de l'accélération de la pesanteur ( $\mathrm{g}$ ) et du débit volumique $(\mathrm{Q})$ sont négligeables devant les contributions de l'inclinaison $(\alpha)$ et des hauteurs d'eau à mi-bassin $(Z$ et $Z_{0}$ ). L'incertitude élargie est globalement comprise entre $18 \%$ et $30 \%$ toutes configurations confondues, elle augmente avec le débit volumique (Q). La contribution de l'inclinaison $(\alpha)$ diminue avec la pente alors que les contributions des hauteurs d'eau $\left(Z\right.$ et $\left.Z_{0}\right)$ augmentent. Le paramètre le plus influent est la hauteur d'eau à mi-bassin du bassin amont $\left(Z_{0}\right)$ qui peut représenter jusqu'à $75 \%$ de la contribution globale.

La connaissance de l'incertitude de mesure sur le coefficient de débit permet tout d'abord d'intervenir dans notre protocole de mesure pour chercher à l'améliorer et à réduire par la même l'incertitude élargie. Elle donne une garantie aux bureaux d'études, effectuant le dimensionnement de ces ouvrages, sur l'évolution du coefficient de débit en fonction des caractéristiques géométriques et hydrauliques des passes à poissons. Ainsi, cette approche en laboratoire permet d'envisager une prise en compte plus juste des paramètres à prendre en considération pour le dimensionnement afin d'éviter des actions correctives une fois l'ouvrage construit.

Les perspectives de cette démarche dans notre laboratoire de recherche sont tout d'abord l'amélioration de la démarche pour mesurer ce coefficient afin d'essayer de 
réduire davantage l'incertitude élargie. Ensuite une généralisation de la démarche sera entreprise sur des mesures ponctuelles de force, de pression, de vitesse, puis à des mesures de champs de vitesse, de température ou de concentration. Les applications étudiées à partir de ces mesures sont les écoulements aérodynamique et hydrodynamique et, dans notre cas plus précisément, les écoulements naturels et environnementaux.

\section{Références}

1. Bell M. (1986) Fisheries Handbook of engineering requirements and biological criteria - Fish Passage Development and Evaluation Program, US Army Corps of Engineers, North Pacific Division Portland, Oregon, 290 p.

2. BfG (2010) Herstellung der Durchgängigkeit an Staustufen von BundeswasserstraßenFischökologische Einstufung der Dringlichkeit von Maßnahmen für den Fischaufstieg (Restoring ecological connectivity along regulated inland waterways - prioritization of fish passage from a fish ecological perspective), BfG report no. 1697.

3CETMEF, VNF (2008) Guide des passes à poissons

4. DWA (2010) Fischaufstiegsanlagen und fischpassierbare Bauwerke - Gestaltung, Bemessung, Qualitätssicherung (Fishways and fish passable buildings: design, dimension and quality assurance), draft Code of Practice M-509.

5.GHAAPPE (2006) Guide technique pour la conception des passes à poissons naturelles, rapport GHAAPPE/ONEMA RA.06.05

6. GHAAPPE (2008) Guide pour la conception de prises d'eau "ichtyocompatibles" pour les petites centrales hydroélectriques, rapport GHAAPPE/ONEMA RA.08.04

7. Gough P., Philisen P., Schollema P.P., Wanningen H. (2012) From sea to source: international guidance for the restoration of fish highways. Wanningen, $\mathrm{H}$. (Eds.)

8.Larinier, M., Travade, F. Porcher, J.P. (2002) Fishways: biological basis, design criteria and monitoring. Bull. Fr. Pêche Pisc. 364, 1-208. 